\title{
International Particle Physics Masterclasses - Bringing LHC data into the Classroom
}

\author{
Farid Ouid-Saada ${ }^{1}$ \\ Department of Physics, University of Oslo \\ P.O.Box 1048 Blidern, 0316 OSLO, Norway \\ E-mail: farid.ould-saadalfys.uio.no
}

The International Particle Physics Outreach Group (IPPOG) has developed an educational activity that brings the excitement of cutting-edge particle physics research into the classroom. Each year, since 2005, thousands of high school students in many countries all over the world come to nearby universities or research centres for one day in order to unravel the mysteries of particle physics and be "scientists for a day". In 2012, 10000 students from 148 institutions in 31 countries took part in the popular event over 4 weeks. Lectures from active scientists give insight on topics and methods of fundamental research on the building blocks of matter and the forces between them, enabling the students to perform measurements on real data from particle physics experiments themselves. The last two years featured the use of LHC data from the ALICE, ATLAS and CMS experiments. We will describe the methodology employed for the IPPOG International Masterclasses, summarise the measurements performed and report on the impact of the day on young students. We will show how you (the particle physicist) can become involved in this activity and develop your own initiatives based around the samples of LHC data and associated tools.

36th International Conference on High Energy Physics

July 4-11, 2012

Melbourne, Australia

${ }^{1}$ Speaker 


\section{Introduction}

The experiments at the Large Hadron Collider (LHC) have already collected a large amount of data and have started to produce interesting new results. The International Masterclasses (IMC) for high school students aged 15-18 have, for the past two years, featured a sample of these fresh LHC data from the ALICE, ATLAS and CMS experiments. Educational material, methods and analysis tools have been developed to accommodate the new data. The emphasis goes from sharing the enthusiasm of our field to explaining to the teachers and young students how things really work, by active participation through involvement in "do-it-yourself" measurements and searches.

The International Particle Physics Outreach Group - IPPOG [1] is a network of scientists, researchers, science educators and communication specialists engaged in informal science education and outreach for particle physics. The International Masterclasses started in 2005 with the analysis of real data from the DELPHI and OPAL experiments at the LEP collider at CERN, inspired by similar activities that were taking place in the UK. The main idea was to allow students to experience how scientists investigate nature by doing it themselves. The students and their teachers go to a nearby university or research centre for a day. Here, students and teachers get insight into topics and methods of basic research on the fundamentals of matter and forces. They then perform measurements on real data from particle physics experiments at CERN.

Students quickly master real event-display programs, software tools and analysis methods. They first practice particle identification by exploiting the characteristic signals left by particles in various sub-detector elements; electrons, muons, photons and jets of particles are then recognised. The students go on to perform event selection and categorisation, and then measure various properties of some known particles, such as the $\mathrm{W}$ and $\mathrm{Z}$ weak gauge bosons and a number of hadrons $\left(J / \psi, Y, \Lambda, K_{s}\right)$.

They also study complex particle properties, such as the quark-gluon structure of the proton (that they come to understand is not just the simple view of uud quarks) through the fraction of $\mathrm{W}^{+}$and $\mathrm{W}^{-}$events in CMS/ATLAS data, and learn how the concept of invariant mass can be used to identify and measure masses and widths of short-lived particles. This builds upon familiar concepts such as conservation laws. Finally, the invariant mass concept is applied to look for new particles (either simulated or real).

At the end of each day, using the same tools as our international research collaborations, the participants join in a videoconference with CERN or Fermilab for discussion and combination of their results. The resulting combinations are then compared to recent results published by the collaborations.

\section{Examples of measurements}

\subsection{ATLAS}

The Universities of Dresden and Oslo [2] have developed Masterclass measurements based on ATLAS data.

The "Z-path" [3] exploits the invariant mass concept as a tool for identifying known short-lived particles and to search for and discover new ones. An ATLAS data sample of 10000 events comprises 9000 real events (dileptons from Z, J/ $\psi$, Y, Drell-Yan, and other QCD and W data), and 1000 simulated dilepton $Z$ ' particles at a mass of $1 \mathrm{TeV}$. Each pair of students uses the 
HYPATIA [4] event display software to examine a subset of 50 mixed events. Particles are then identified, dilepton $\left(\mathrm{e}^{+} \mathrm{e}^{-}\right.$or $\left.\mu^{+} \mu^{-}\right)$events selected and invariant masses calculated. A web-based application [5] provides a plotting tool as well as a facility for upload and combination of results (Figure 1). The students then discuss with physicists the measured masses and widths of the known resonances, make a comparison between electrons and muons and compare with results published by ATLAS. They also "discover" a new particle with a mass around $1 \mathrm{TeV}-$ the simulated Z'. Such a new neutral gauge boson is supposed to mediate a hypothetical new weak interaction predicted by some theories beyond the Standard Model.

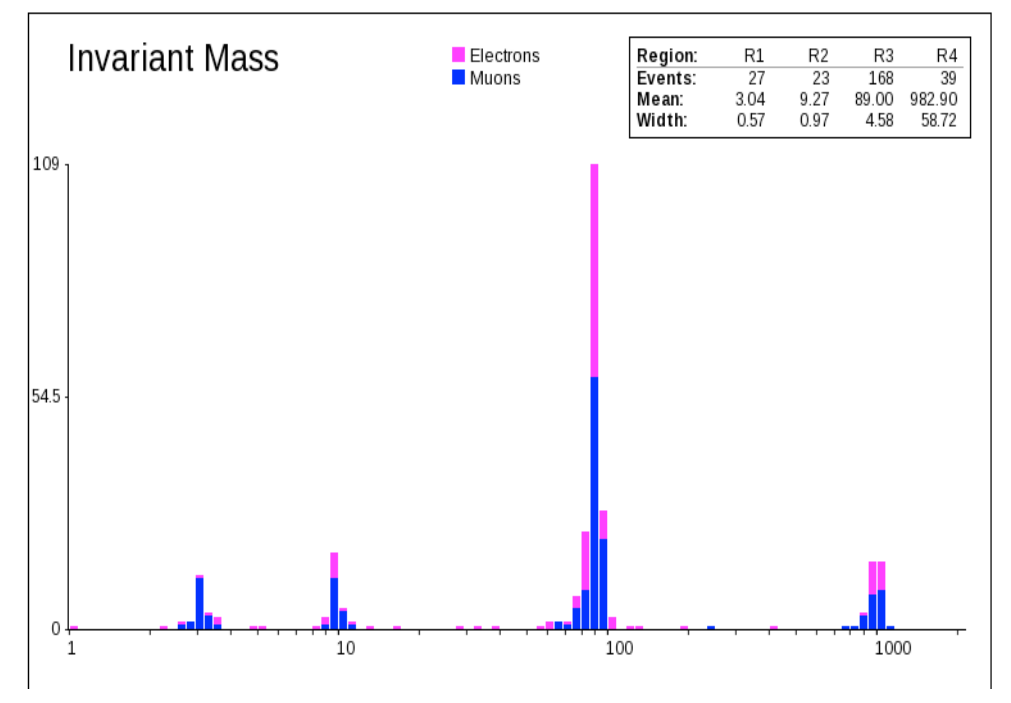

Figure 1. ATLAS dilepton data as analysed by students, showing the $J / \psi, Y$ and $Z$ resonances (data) as well as a simulated $Z^{\prime}$ peak at $1 \mathrm{TeV}$.

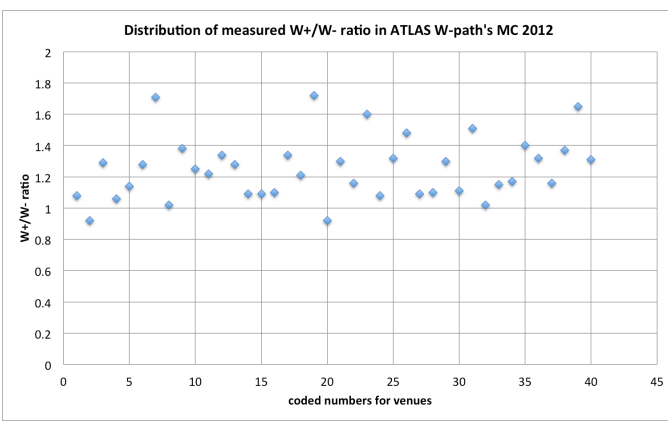

Figure 2. The $W^{+} / W$ ratio measured by the students is close to that expected from this data sample, and far from the prediction that they make using the simple und model of the proton.

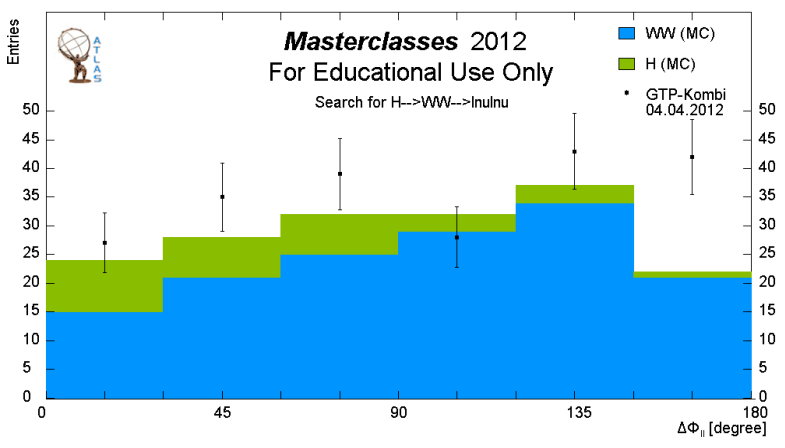

Figure 3. Distribution of the angle between the 2 leptons $\Delta \Phi_{l l}$ in the transverse plane for $W W$ and $H \rightarrow W W$ events. "GTP-Kombi" corresponds to a combined measurement performed by a few groups of students.

The ATLAS "W-path" [6] makes use of a data sample of 6000 events, mainly consisting of $\mathrm{W} \rightarrow \mathrm{lv}$ events with a high transverse momentum electron or muon and some missing transverse energy/momentum. Some background events (QCD jets, Z, top) and 250 simulated $\mathrm{H} \rightarrow \mathrm{WW}$ are added. The event display program MINERVA [7] is used to identify W bosons. The students measure the fractions of $\mathrm{W}^{+}$and $\mathrm{W}^{-}$events (Figure 2), as well as the angle between leptons from $2 \mathrm{~W}$-bosons in the transverse plane (Figure 3). Students make use of online spreadsheets [8] to document their measurements and from which summary tables and histograms are 
extracted. The data sample made available for student analyses has a $\mathrm{W}^{+} / \mathrm{W}^{-}$ratio of $1.56 \pm 0.17$, very close to the published ATLAS result of $1.52 \pm 0.07$.

\subsection{CMS}

The measurements based on CMS data have mainly been developed by QuarkNet [9], I2U2 [10] and the CMS Education and Outreach team. In the 2012 version of the CMS Masterclass [11], students are asked to study $\mathrm{W}$ and $\mathrm{Z}$ boson candidates using the purely web-based event display software iSpy-online [12]. Students examine and manipulate events in three dimensions to determine lepton flavour (e or $\mu$ ), candidacy $\left(W, Z\right.$, or "zoo") and charge $\left(\mathrm{W}^{+}\right.$or $\left.\mathrm{W}^{-}\right)$. They then find $\mathrm{W}^{+} / \mathrm{W}^{-}$and e $/ \mu$ ratios, as well as reconstructing the $Z$-mass. They find that many of their $Z$ candidates were, in fact, other, lower mass particles: $\mathrm{J} / \psi$ and $\mathrm{Y}$. The recording of events and calculations are done by use of an EditGrid web-based spreadsheet. An example is shown in Figure 4. Students were able to determine that an event contains the decay of a $\mathrm{W}$ with $95 \%$ efficiency. Of the correctly-identified events they were able to determine the charge (from electron or muon) for $92 \%$ of the events (if not, they marked them as "W candidate" with no charge specified). And for those events where they were able to determine the charge, they were able to determine it correctly with $96 \%$ efficiency. This high efficiency leads to the students finding a $\mathrm{W}^{+} / \mathrm{W}^{-}$ratio that is very close to the value in the data, that is in turn very close to the published value (1.43).

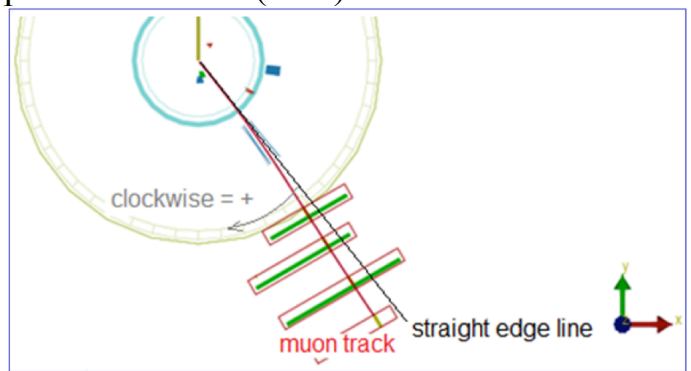

Figure 4. Charge determination and invariant mass (right plot).

\subsection{ALICE}

The students' ALICE analysis tool [13] relies on a simplified version of their ROOT-based event display software. The tool contains $\mathrm{V}_{0}$ and cascade decay finders, allows calculation of invariant masses (Figure 5) as well as the filling of tables and histograms. Students study production of strange particles and count the observed numbers of $K_{s}, \Lambda$ and anti- $\Lambda$; they then compare the yields to Monte Carlo predictions as well as to published results. The goal is, by comparing results from proton collisions and from lead collisions, to observe strangeness enhancement as a telltale signal of the production of the quark-gluon plasma.
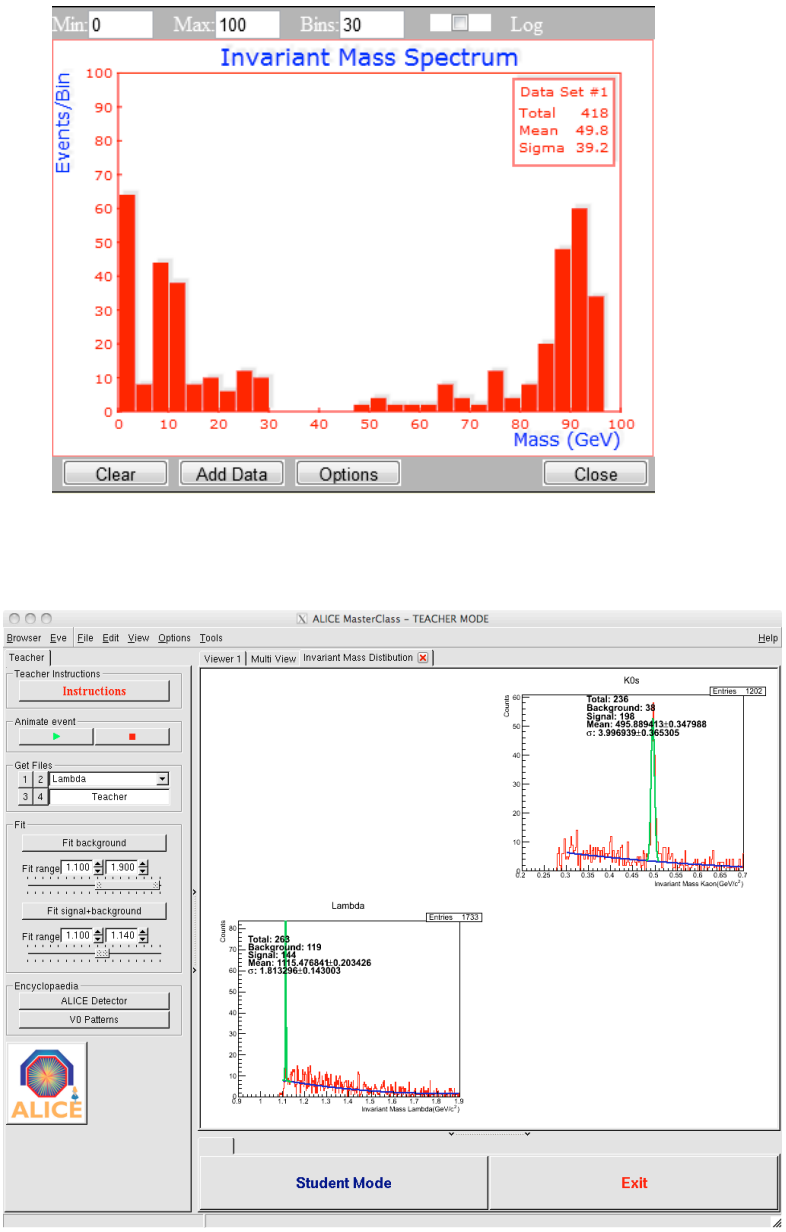

Figure 5. Invariant mass plot for $K_{s}$ and $\Lambda$. 


\section{International Masterclasses 2012 - Statistics and Growth}

The 2012 IPPOG Masterclasses [14] featured an increase in participation compared to 2011 when LHC measurements were first introduced.

Table 1. IMC statistics in 2011 and 2012. VC stands for video-conferences at CERN or Fermilab led by moderators.

\begin{tabular}{|l|c|c|c|c|c|c|c|}
\hline & Days & Countries & Institutes & Students & Moderators & VC & Classes \\
\hline 2011 & 19 & 26 & 99 & 9000 & 15 & 23 & 116 \\
\hline 2012 & 21 & 31 & 118 & 10000 & 21 & 36 & 143 \\
\hline
\end{tabular}

Table 2. IMC Statistics on LHC measurements. In addition, 31 institutes followed the 2012 US program; 13 did the ATLAS Z-measurement and 18 the CMS W\&Z measurement.

\begin{tabular}{|c|c|c|c|c|}
\hline & ALICE & ATLAS W & ATLAS Z & CMS \\
\hline 2011 & 10 & 49 & 31 & 26 \\
\hline 2012 & 13 & 42 & 53 & 35 \\
\hline
\end{tabular}

Surveys are conducted immediately after the Masterclasses in order to assess the whole event and allow an examination of aspects to be improved, simplified or introduced. The results of the 2012 surveys concluded that the IPPOG Masterclasses have been a great success and that interest is growing. Students, teachers and moderators enjoy participating, learn many things and have an increased interest in basic research after the Masterclasses. But there is still room for improvement: the theory is sometimes found rather difficult to grasp; some hands-on measurements have the tendency to be too easy and repetitive. Some students ask for more challenges and to be updated on the research front. They want to "take part in discoveries".

\section{Prospects}

At least 8 more countries have shown interest to join the 2013 IMC and will be added to the 2012 map below: Australia, Cyprus, Georgia, India, Turkey, Romania, Egypt, Palestine.

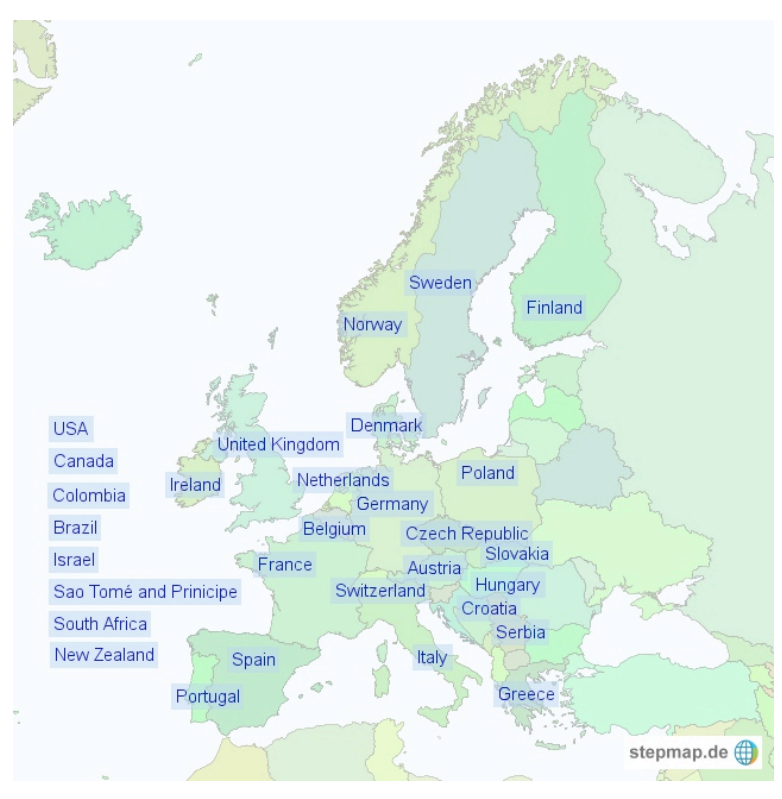


"On-demand" Masterclasses are more and more organised: when and where it suits.

Work is ongoing to improve and converge towards common tools (plotting, uploading, combining, looping over large samples of data). All measurements are being updated and improved. The LHC experiments have recognized the success and potential of the International Masterclasses and have recently approved the release of larger and more exotic data samples. The ATLAS Z-path is extended to apply the invariant mass technique to cover current research highlights: $\mathrm{H} \rightarrow \mathrm{ZZ} \rightarrow 1111, \mathrm{H} \rightarrow \gamma \gamma$. The ATLAS W-path will make use of real WW events. The ATLAS collaboration allows IMC to use $1 \mathrm{fb}^{-1}$ of data to cover these Higgs "searches". The CMS collaboration makes available several Higgs candidates in the mass region of interest in various decay channels for "treasure hunt" activities. Heavy ion collision data will be used more extensively in the coming ALICE measurements. New ideas and concepts are highly welcome. The IPPOG web site hosts a collection of resources [15], including many that are useful for developing Masterclass activities.

\section{References}

[1] International Particle Physics Outreach Group - IPPOG, http://ippog.web.cern.ch, http://facebook.com/IPPOG

[2] LHC@InternationalMasterclasses, http://atlas.physicsmasterclasses.org/en/index.htm

[3] M. Pedersen, F. Ould-Saada, E. Gramstad, M. Bugge, University of Oslo, The ATLAS Z-path, http://atlas.physicsmasterclasses.org/en/zpath.htm

[4] HYPATIA, Ch. Kourkoumelis, D. Fassouliotis, S. Vourakis (University of Athens), D. Vudragovic (Institute of Physics, Belgrade), http://hypatia.phys.uoa.gr

[5] V. Morisbak, M. Pedersen, F. Ould-Saada, University of Oslo, OPloT, http://cernmasterclass.uio.no/OPloT/index.php

[6] K. Jende, M. Kobel, G. Pospiech, U. Bilow, C. Rudolph, University of Dresden, The ATLAS Wpath, http://atlas.physicsmasterclasses.org/en/wpath.htm

[7] MINERVA, T. McLaughlan, M. Stockton, P. Watkins (University of Birmingham), M. Wielers (Rutherford Appleton Laboratory), http://atlas-minerva.web.cern.ch/atlas-minerva

[8] EditGrid, http://www.editgrid.com/user/masterclass/Analysis 2012

[9] QuarkNet, http://quarknet.fnal.gov

[10] I2U2, http://www.i2u2.org

[11]K. Cecire et al, CMS Masterclass W/Z Measurement, http://cms.physicsmasterclasses.org/pages/cmswz.html

[12] iSpy-online, M. Hategan, P. Nguyen, T. McCauley, i2u2 and QuarkNet collaborations, http://www.i2u2.org/elab/cms/event-display

[13] P.R. Debski, P.Foka, D.Hatzifotiadou, B.Hippolyte, A. Maire, M. Tadel. Proceedings of the 7th International Conference Hands-on Science Bridging the Science and Society gap, July 25 - 31, 2010, Rethymno - Greece. http://aliceinfo.cern.ch/public/MasterCL/MasterClassWebpage.html

[14] International Masterclasses, www.physicsmasterclasses.org

[15]IPPOG database, http://ippog.web.cern.ch/resources 